\title{
О НЕПРЯМОЛИНЕЙНОМ РАСПРОСТРАНЕНИИ СВЕТА В ИНТЕГРАЛЬНОЙ ФОТОУПРУГОСТИ ТЕЛ ВРАЩЕНИЯ
}

\section{1. Введение}

Обычно принято считать, что в фотоупругих моделях свет распространяется прямолинейно. На самом же деле траектории световых лучей в таких моделях несколько отклоняются от прямолинейных в связи с неравномерным распределением компонентов показателя преломления. Этот вопрос применительно к двухмерным моделям изучался в $\left[{ }^{1-5}\right]$, а применительно к голографической интерферометрии фазовых объектов - в $\left[{ }^{6,7}\right]$. В $\left[{ }^{8}\right]$ рассматривалась возможность использования эффекта фотоупругости для создания градиента показателя преломления в оптических волокнах. Показано, что измерение отклонения световых лучей позволяет определить осесимметричное скалярное поле показателя преломления $\left[{ }^{9-11}\right]$.

Отклонение светового луча от прямой тем больше, чем больше градиент показателя преломления в перпендикулярном лучу направлении и чем длиннее путь этого луча в неоднородной среде. С этой точки зрения представляет интерес исследовать отклонение световых лучей на моделях интегральной фотоупругости $\left[{ }^{12,13}\right]$, напряжения в которых определяются на основе интегральных оптических величин, получаемых при просвечивании целой модели в полярископе. Постановка этой задачи даң̨а в $\left[{ }^{14}\right]$. Целью настоящей статьи является исследование некоторых вопросов, связанных с влиянием отклонения световых лучей на экспериментальные данные интегральной фотоупругости тел вращения.

\section{2. Вычисление траекторий световых лучей в осесимметричном поле показателя преломления}

Рассмотрим просвечивание тела вращения в плоскости симметрии, перпендикулярной его оси $z$ (рис. 1). В данном случае тело оптически характеризуется распределением трех главных показателей преломления $n_{r}(r), n_{\Theta}(r)$ и $n_{z}(r)$.

Траектория светового луча с колебаниями, параллельными оси $z$, определяется показателем преломления $n_{z}$. Так как распределение последнего осесимметрично, здесь применима формула Бугера $\left[{ }^{11,15}\right]$ (рис. 2)

$$
n r \sin \varphi=\mathrm{const}=c,
$$

откуда следует формула для вычисления траектории светового луча:

$$
\Theta=\Theta_{0}+c \int_{R}^{r} d r / r \sqrt{n^{2} r^{2}-c^{2}}
$$

где $n=n(r)$. Поэтому интеграл (2) в замкнутом виде, как правило, не выражается. Для численного интегрирования формула (2) неудобна 


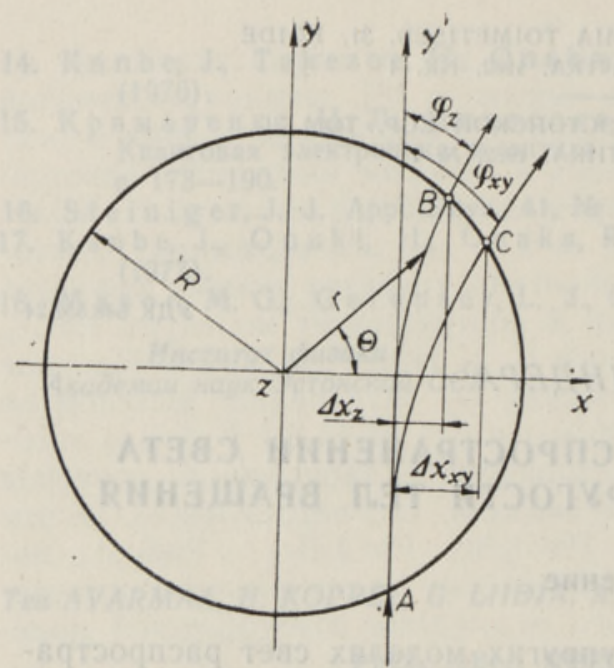

$$
n_{x}=n_{r} \cos ^{2} \Theta+n_{\Theta} \sin ^{2} \Theta .
$$

Из формулы (3) видно, что распределение показателя преломления $n_{x}$ не является осесимметричным, хотя $n_{r}$ и $n_{\Theta}-$ функции осесимметричные. Следовательно, для светового луча с колебаниями в плоскости $x y$ формулу Бугера использовать нельзя.

Общее уравнение траектории светового луча в плоскости $x y$ имеет вид $\left[{ }^{11}\right]$

$$
n\left(d^{2} x / d y^{2}\right)=\left[1+(d x / d y)^{2}\right][\partial n / \partial x-(\partial x / \partial y)(\partial n / \partial y)] .
$$

Вычисления показывают, что в случае фотоупругости имеет место неравенство

$$
d x / d y<10^{-2}
$$

T. e.

$$
(d x / d y)^{2} \ll 1 .
$$

Следовательно, членом $(d x / d y)^{2}$ в правой части уравнения (4) можно пренебречь.

В общем случае не исключено, что градиент показателя преломления в направлении оси $y$ значительно больше, чем в направлении оси $x$. Однако, учитывая неравенство (5), в отношении (4) можно пренебречь также членом $(\partial x / \partial y)(\partial n / \partial y)$. Тогда вместо уравнения (4) получим уравнение, обычно используемое в расчетах

$$
d^{2} x / d y^{2}=(1 / n)(\partial n / \partial x) .
$$

Так как изменение показателя преломления в фотоупругих моделях обычно не превышает $10^{-3}$, уравнение (7) можно записать в виде

$$
d^{2} x / d y^{2}=\left(1 / n_{0}\right)(\partial n / \partial x),
$$

где $n_{0}$ - показатель преломления ненапряженного материала. Напомним, что в уравнении (8) для световых лучей с колебаниями, параллельными оси $z, n=n_{z}$, а для лучей с колебаниями в плоскости $x y-n=n_{x}$.

Таким образом, в общем случае световой луч, входя в осесимметричную модель в точке * $A$ (рис. 1$)$, разделяется на два луча с коле-

* Предполагается, что модель находится в иммерсионной ванне, и световой луч в точках входа в модель и выхода из нее не преломляется. 


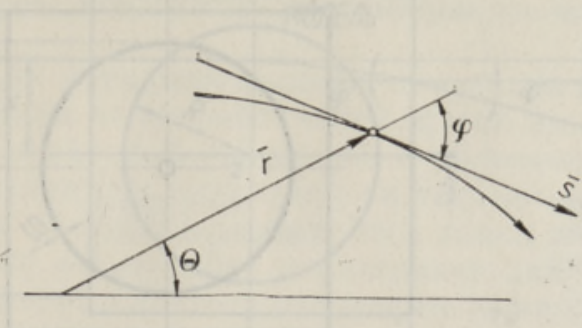

Рис. 2. К объяснению формулы Бугера.

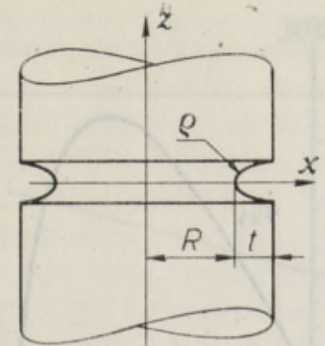

Рис. 3. Геометрия первой модели.

баниями, параллельными оси $z$ и плоскости $x y$ соответственно. Из модели выходят два луча в точках $B$ и $C$, которые образуют с первоначальным направлением луча углы $\varphi_{z}$ и $\varphi_{x y}$.

Из уравнения (8) следует, что угловое отклонение световых лучей определяется формулой

$$
\varphi=d x / d y=\varphi_{0}+\left(1 / n_{0}\right) \int(\partial n / \partial x) d y,
$$

а линейное $\Delta x-$ формулой

$$
\Delta x=\Delta x_{0}+\int \varphi(y) d y .
$$

Так как линейное отклонение $\Delta x$ заведомо весьма мало, траекторию луча приближенно можно считать дугой круга. В последнем случае имеем

$$
\Delta x \cong(1 / 2 \varphi) \sqrt{R^{2}-x^{2}},
$$

где $\varphi$ - полное угловое отклонение луча при прохождении модели.

Чтобы получить представление о порядке величины отклонения световых лучей, рассмотрим две фотоупругие модели.

Первая - цилиндрический стержень с гиперболической вырезкой при растяжении (рис. 3). Распределение напряжений для случая $R / \varrho=5, t / R=1 / 3$ вычисляли по формулам Нейбера $\left[{ }^{16}\right]$, а показатели преломления - по формулам Максвелла-Неймана

$$
\begin{aligned}
& n_{r}=n_{0}+C_{1} \sigma_{r}+C_{2}\left(\sigma_{\Theta}+\sigma_{z}\right), \\
& n_{\Theta}=n_{0}+C_{1} \sigma_{\Theta}+C_{2}\left(\sigma_{z}+\sigma_{r}\right), \\
& n_{z}=n_{0}+C_{1} \sigma_{z}+C_{2}\left(\sigma_{r}+\sigma_{\Theta}\right),
\end{aligned}
$$

где $C_{1}, C_{2}$ - фотоупругие постоянные. Считалось, что среднее осевое напряжение равняется 5 МПа и что модель сделана из материала «Palatal P6». В последнем случае имеем $C_{1}=-26 \cdot 10^{-12} \Pi a^{-1}, C_{2}=$ $=-55 \cdot 10^{-12} \Pi a^{-1}$ для $\lambda=514,5$ нм [5]. Зависимость углового отклонения $\varphi_{x y}$ и $\varphi_{z}$ от координаты падающего светового луча $x$ показана на рис. 4 сплошными кривыми. Максимальному угловому отклонению 3 мрад соответствует линейное отклонение $\Delta x_{x y}=0,0015 R$.

Вторая модель - цилиндрический стержень из кварцевого стекла с закалочными напряжениями

$$
\begin{aligned}
& \sigma_{r}=\sigma_{0}\left[1-(r / R)^{2}\right] / 2, \\
& \sigma_{\Theta}=\sigma_{0}\left[1-3(r / R)^{2}\right] / 2, \\
& \sigma_{z}=\sigma_{0}\left[1-2(r / R)^{2}\right],
\end{aligned}
$$

где $\sigma_{0}$ - осевое напряжение при $r=0$. Фотоупругие постоянные в дан- 


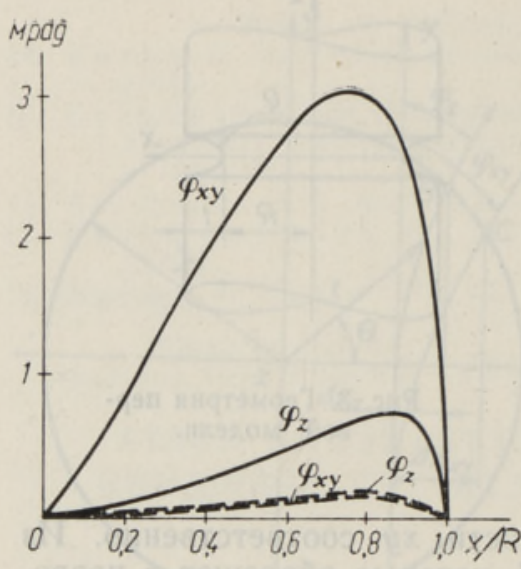

Рис. 4. Отклонение световых лучей в цилиндрическом стержне с гиперболической вырезкой (-) и в цилиндрическом стержне с закалочными напряжениями $(---)$.
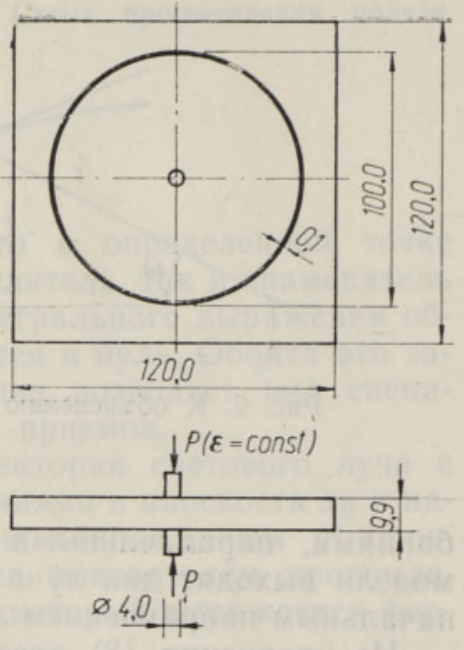

Рис. 5. Геометрия модели.

ном случае следующие $\left[{ }^{17}\right]: \quad C_{1}=-0,69 \cdot 10^{-12} \quad \Pi a^{-1}, \quad C_{2}=$ $=-4,27 \cdot 10^{-12} \Pi a^{-1}$. Угловые отклонения показаны на рис. 4 пунктирными кривыми. Максимальное линейное отклонение составляет

$$
\Delta x_{x y}=0,00018 R \text {. }
$$

Величина $\varphi$ определялась численным интегрированием формулы (9), а $\Delta x-$ с помощью формулы (11).

Эти примеры показывают, что степень отклонения световых лучей в зависимости от поляризации может быть различной. Судя по сильной концентрации напряжений в материале высокой оптической чувствительности (первая модель), можно ожидать, что максимальное угловое отклонение световых лучей в фотоупругих моделях составляет несколько мрад.

\section{3. Экспериментальное исследование отклонения световых лучей}

Отклонение световых лучей исследовали в осесимметричном поле напряжений с высоким градиентом последних. Изготовленную из «Palatal P6» (BASF) круглую пластинку диаметром 100,0 мм и толщиной 9,9 мм нагружали в центре двумя концентрированными силами. Силы передавались пластинке через медные цилиндры диаметром 4,0 мм. Из того же материала изготовили квадратную пластинку с длиной стороны 120 мм и просверлили в ней круглое отверстие, радиус которого превышал радиус круглой пластинки на 0,1 мм. Круглую пластинку вставили в отверстие квадратной, предварительно смочив края обеих пластинок иммерсионной жидкостью (Dow Corning Diffusion Pump Fluid № 704, $n=$ $=1,553$ для $\lambda=633$ нм), исключив тем самым возможность преломления света по краям круглой пластинки. Геометрия модели показана на рис. 5 .

Чтобы избежать диффузии света при входе в модель и при выходе из нее, на соответствующие поверхности модели были прикреплены плоскопараллельные стеклянные пластинки, которые отделял от них слой иммерсионной жидкости.

Модель просвечивали в срединной поверхности согласно схеме, показанной на рис. 6 , и измеряли линейное отклонение света $\Delta x^{\prime}$ на экране. 


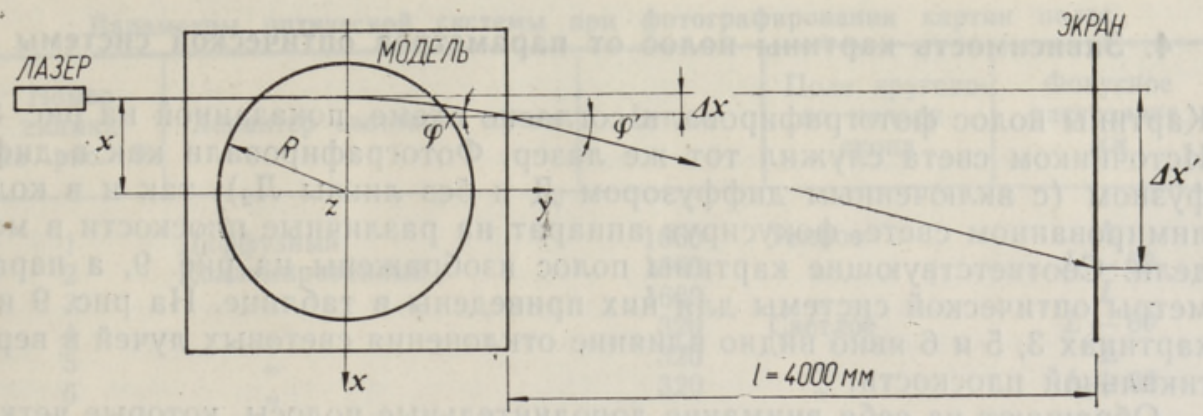

Рис. 6. Схема измерения отклоненгя световых лучей.
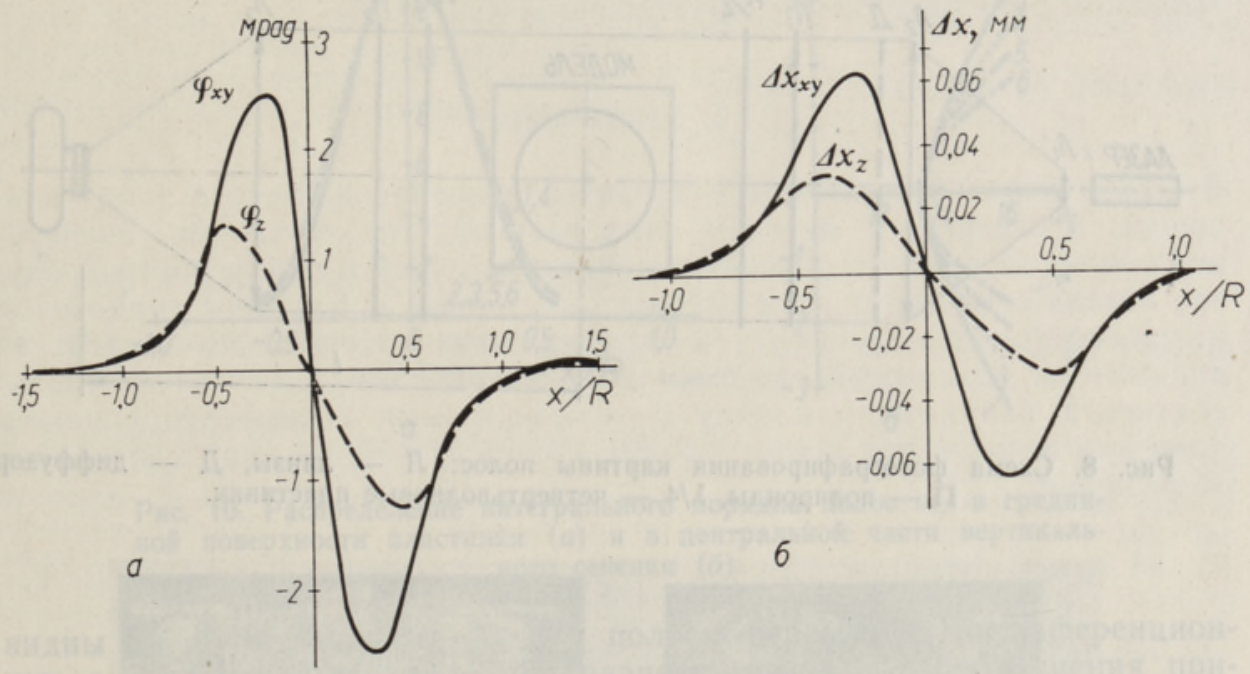

6

Рис. 7. Графики отклонения световых лучей: угловые (a) и линейные (б).

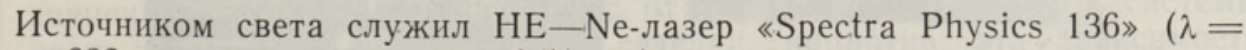
$=633$ нм, диаметр пучка - 0,51 мм).

На экране пятно лазерного луча получалось диаметром около 10 мм. Его положение удавалось определить с помощью установленного перед экраном светофильтра с точностью до 0,3 мм. Модель до и после приложения нагрузки дважды сканировали линейно поляризованным лазерным лучом с колебаниями как в плоскости модели, так и в перпендикулярном к ней направлении.

На основе экспериментально измеренных величин $\Delta x^{\prime}$ при сканировании модели по координате $x$ вычислялось угловое отклонение $\varphi$ по формуле

$$
\varphi \cong \varphi^{\prime} / n_{0},
$$

где

$$
\varphi^{\prime} \cong \Delta x^{\prime} / l .
$$

Зависимость углов $\varphi_{x y}$ и $\varphi_{z}$ от безразмерной координаты луча $x / b$ $(b-$ толщина модели) показана на рис. $7, a$, откуда видно, что максимальное отклонение лучей с колебаниями в плоскости $x y$ примерно вдвое больше, чем лучей с колебаниями, параллельными оси $z$. Максимумы графиков $\varphi_{x y}$ и $\varphi_{z}$ смещены относительно друг друга.

Графики линейных отклонений $\Delta x_{x y}$ и $\Delta x_{z}$ показаны на рис. $7, б$. 


\section{4. Зависимость картины полос от параметров оптической системы}

Картины полос фотографировали согласно схеме, показанной на рис. 8. Источником света служил тот же лазер. Фотографировали как в диффузном (с включенным диффузором Д и без линзы $Л_{2}$ ), так и в коллимированном свете, фокусируя аппарат на различные плоскости в модели. Соответствующие картины полос изображены на рис. 9, а параметры оптической системы для них приведены в таблице. На рис. 9 на картинах 3,5 и 6 явно видно влияние отклонения световых лучей в вертикальной плоскости.

Обращают на себя внимание дополнительные полосы, которые четко

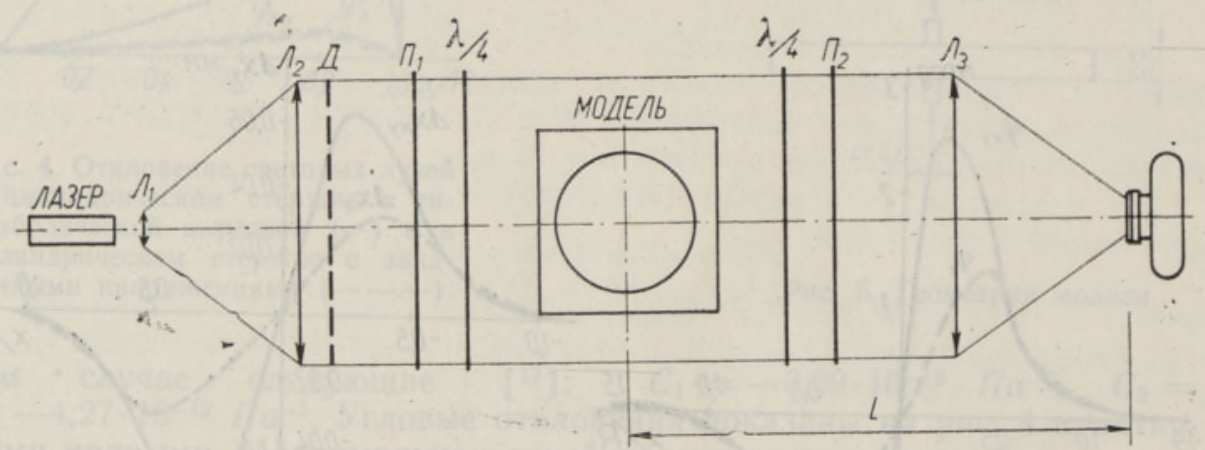

Рис. 8. Схема фотографирования картины полос: Л - линзы, Д - диффузор, П - поляронды, $\lambda / 4-$ четвертьволновые пластинки.

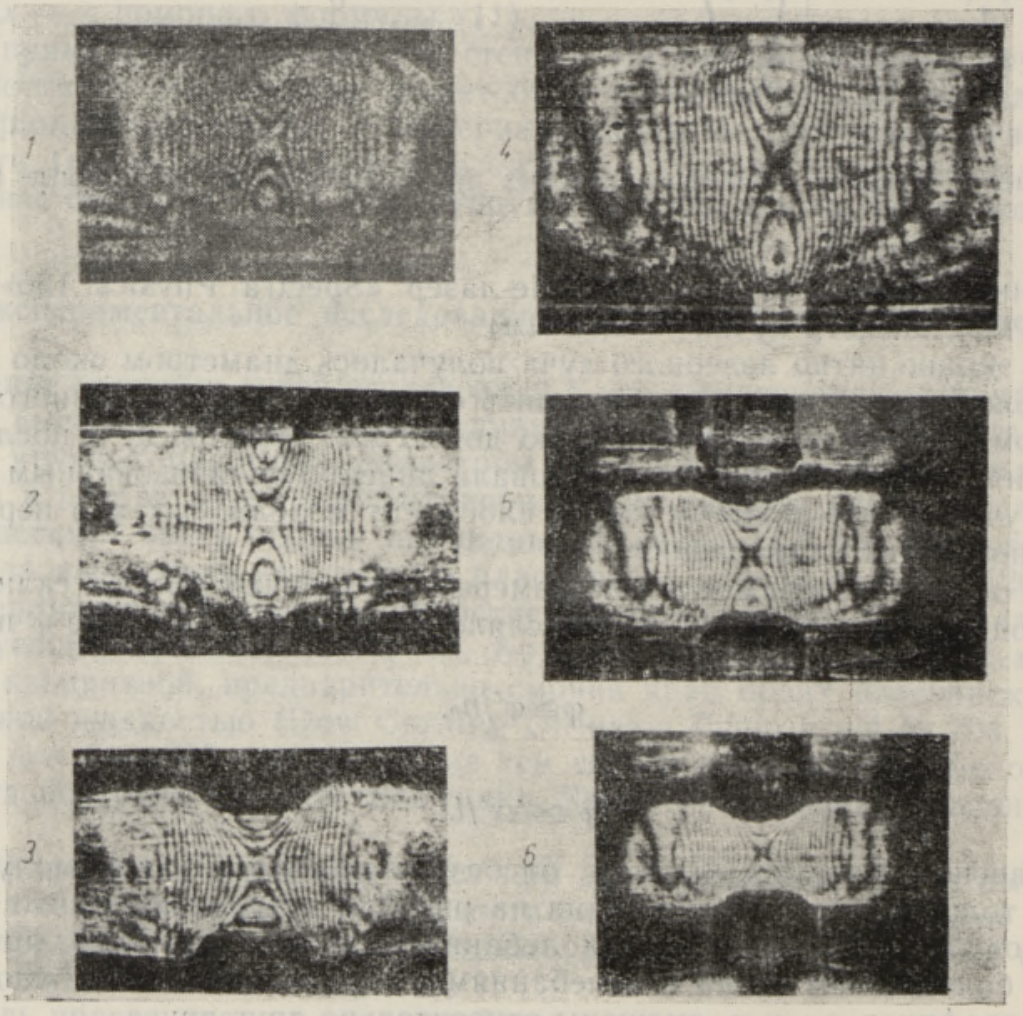

Рис. 9. Картины полос (см. таблицу). 
Параметры оптической системы при фотографировании картин полос

\begin{tabular}{|c|c|c|c|c|}
\hline $\begin{array}{c}\text { Номер } \\
\text { снимка } \\
\text { на рис. } 9\end{array}$ & Характер светового пучка & $L ; M M$ & $\begin{array}{c}\text { Поле кругово- } \\
\text { го поляри- } \\
\text { скопа }\end{array}$ & $\begin{array}{c}\text { Фокусное } \\
\text { расстояние, } \\
\text { мм }\end{array}$ \\
\hline $\begin{array}{l}1 \\
2 \\
3 \\
4 \\
5 \\
6\end{array}$ & $\begin{array}{c}\text { Диффузный } \\
\text { Коллимированный } \\
\text { " } \\
, \\
,\end{array}$ & $\begin{array}{r}1660 \\
1660 \\
1660 \\
320 \\
320 \\
320\end{array}$ & $\begin{array}{c}\text { Темное } \\
\text { " } \\
\text { Светлое } \\
, " \\
,\end{array}$ & $\begin{array}{l}L \frac{L}{L} 60 \\
L \frac{L}{L} 60 \\
L+30\end{array}$ \\
\hline
\end{tabular}

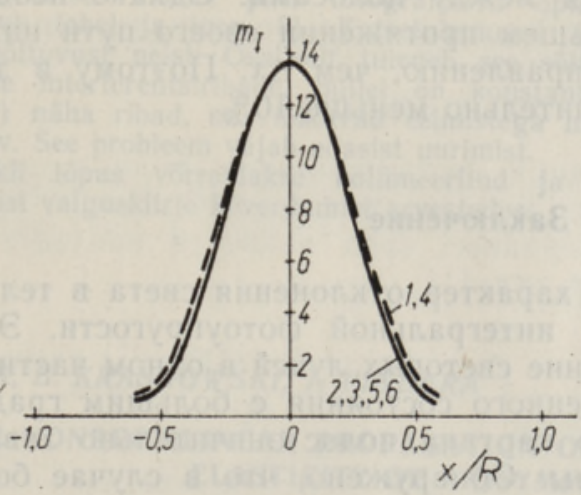

a

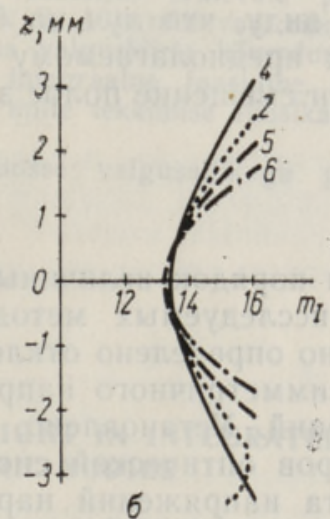

6

Рис. 10. Распределение интегрального порядка полос $m_{I}$ в срединной поверхности пластинки $(a)$ и в центральной части вертикального сечения (б).

видны на фотографиях 4-6. Эти полосы пересекают интерференционные с постоянной интегральной разностью фаз. Для объяснения природы новых полос требуются дальнейшие исследования.

На рис. 10 показано распределение порядка изображенных на рис. 9 интегральных полос. Видно, что если в срединной поверхности модели зависимость распределения полос от параметров оптической системы очень мала, то в вертикальном сечении, напротив, эта зависимость весьма значительна. В этом определенную роль играет отклонение световых лучей.

Различие оптических явлений при использовании коллимированного и диффузного света заключается в следующем. В первом случае пучок света входит в модель параллельно оптической оси полярископа (рис. $11, a)$. В модели свет отклоняется, и выходящие из нее световые лучи образуют больший или меньший угол с первоначальным направлением. В зависимости от апертуры системы в некоторых областях мо-

Рис. 11. Траектории светового луча в случае коллимированного $(a)$ и диффузного (б) источников света.

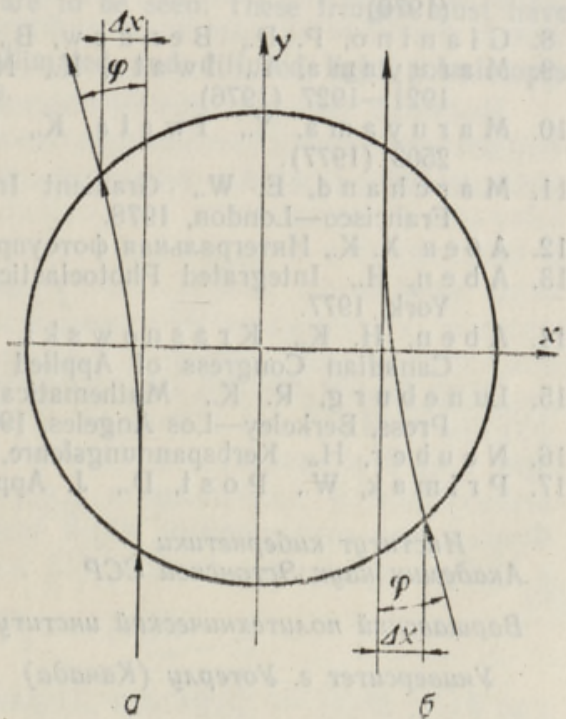


дели картина интерференции может пропадать, аналогично случаю, рассмотренному в $\left[^{1}\right]$.

Во втором случае оптическая система собирает световые лучи, параллельные ее оптической оси (рис. 11,б). Эти лучи входят в модель под некоторым углом к оси системы.

В обоих случаях полосы могут смещаться из-за линейного отклонения $\Delta x$, а также из-за того, что траектория луча в модели отличается от предполагаемой.

В проведенном эксперименте максимальное линейное отклонение света не превышало 0,06 мм при расстоянии между полосами 0,6 мм. Следовательно, максимальное смещение полос может в данном случае составлять $10 \%$ от расстояния между полосами. Однако необходимо иметь в виду, что луч на бо́льшем протяжении своего пути находится ближе к предполагаемому направлению, чем $\Delta x$. Поэтому в действительности смещение полос значительно меньше $10 \%$.

\section{5. Заключение}

Выявлен порядок величины и характер отклонения света в телах вращения, исследуемых методом интегральной фотоупругости. Экспериментально определено отклонение световых лучей в одном частном случае осесимметричного напряженного состояния с большим градиентом напряжений. Установлено, что картина полос значительно зависит от параметров оптической системы. Обнаружено, что в случае большого градиента напряжений наряду с интегральными интерференционными полосами появляются новые полосы, природа которых еще ждет своего объяснения.

\section{ЛИТЕРА Т У РА}

1. Бокш те йн М. Ф., Ж. техн. физ., 19, № 10, 1103-1106 (1949).

2. P in der a, J. T., Rozpr. Inż., 3, № 1, 109-176 (1955).

3. Hecker, F. W., Pindera, J. T., VDI-Ber,, № 313, 745-754 (1978).

4. Hecker, F. W., Ke p i c h, T. Y., P i nder a, J. T., In: Proc. Eighth All-Union Conf. on Photoelasticity, Tallinn, 1979, 1, p. 117-123.

5. Pindera, J. T., Hecker, F. W., K ras nowski, B. R., In: Proc. Eighth Canadian Congress of Applied Mechanics, Moncton, 1981, 2, p. 517-518.

6. V e s t, C. M., Appl. Opt., 14, № 7, 1601-1606 (1975).

7. Гинзбург В. М., Филенко Ю. И., Ж. техн. физ., 40, № 10, 2217-2220 (1970).

8. G i a n i no, P. D., B e nd o w, B., Appl, Opt., 20, № 3, 430-434 (1981).

9. M a ruy am a, Y., I w at a, K., N a g a ta, R., Jap. J. Appl. Phys., 15, № 10 , $1921-1927$ (1976).

10. M a ruy a ma, Y., I w a ta, K., $\mathrm{N}$ a g a ta, R., Appl. Opt., 16, № 9, 25002503 (1977).

11. M a r chand, E. W., Gradient Index Optics, Academic Press, New York-San Francisco-London, 1978.

12. А б е н Х. К., Интегральная фотоупругость, Таллин, «Валгус», 1975.

13. A b e n, H., Integrated Photoelasticity, McGraw-Hill Intern. Book Company, New York, 1977.

14. Aben, H. K., Krasnowski, B. R., P inder a, J. T., In: Proc. Eighth Canadian Congress of Applied Mechanics, Moncton, 1981, 2, 519-520.

15. Luneburg, R. K., Mathematical Theory of Optics, University of California Press, Berkeley-Los Angeles, 1966.

16. N e uber, H., Kerbspannungslehre, Verlag von Julius Springer, Berlin, 1937.

17. Prima k, W., Post, D., J. Appl. Phys., 30, № 5, 779-788 (1959).

Институт кибернетики

Академии наук Эстонской ССР

Вариавский политехнический институт
Поступила в редакцию $5 / \mathrm{X} 1981$

Университет е. Уотерлу (Канада) 


\section{VALGUSE MITTESIRGJOONELISEST LEVIMISEST PÖORDKEHADE INTEGRAALSE FOTOELASTSUSMEETODI KASUTAMISEL}

On näidatud, et optilise anisotroopia telgsümmeetrilise jaotusega pöördkehas kirjeldab valguskiire trajektoori Bouguer' valem vaid juhul, kui valgusvônkumiste suund on paralleelne keha teljega. Telje risttasapinnas toimuvate võnkumiste puhul Bouguer' valem ei kehti ning tuleb kasutada üldisi valemeid (4), (7) vôi (8). Ligikaudselt avaldub valguskiire pöördumine valemiga (9) ning lineaarne kõrvalekalle valemiga (10). On arvutatud valguskiire kõrvalekalded kahel erijuhul (joon. 4).

Eksperimentaalselt on uuritud valguskiire kõverdumist tsentris vastassuunaliste koondatud jõududega koormatud ringikujulises plaadis (joon. 5) vastavalt joonisel 6 oleva!e skeemile. Katsetulemused on toodud joonisel 7 .

Integraalset ribade pilti fotografeeriti optilise süsteemi erinevate parameetrite puhul (vt. tabel ja joon. 8). Katsetulemused (joon. 9, 10) näitavad ribade jaotuse olulist sõltuvust neist. Osaliselt tuleneb see sõltuvus valguskiirte kõverdumisest.

Peale interferentsiribade, millel on konstantne integraalne faasinihe, on fotodel (joon. 9) näha ribad, mis lôikuvad eelmistega ning mille tekkimise füüsikaline põhjus on erinev. See probleem vajab edasist uurimist.

Artikli lõpus võrreldakse kollimeeritud ja difuusse valgusallikaga polariskoobi kasutamist valguskiirte kõverdumist arvestades.

\section{H. ABEN, B. KRASNOWSKI, J. PINDERA}

\section{ON THE NONRECTILINEAR PROPAGATION OF LIGHT IN INTEGRATED PHOTO- ELASTICITY OF AXISYMMETRIC BODIES}

It is shown that in an axisymmetric refractive index tensor field the Bouguer' formula describes the ray trajectory only if the light vibrations are parallel to the axis of symmetry. If light vibrations are in the plane which is perpendicular to the axis of symmetry, one has to use the general Eq. (4) or its simplifications (7) or (8). The angular deflection of the light ray can approximately be determined through Eq. (9) and the linear deflection through Eq. (10). The deflections of the light rays in two particular cases have been calculated (Fig. 4).

Deflection of light in a circular plate loaded at the centre with opposite concentrated loads (Fig. 5) has been studied experimentally using a setup given in Fig. 6. The results are shown in Fig. 7.

Integral fringe patterns were photographed with different parameters of the optical system (collimated or diffused light, dark-field or light-field polariscope, different object planes, see Table and Fig. 8). Fringe patterns are shown in Fig. 9. It is to be seen(Figs. 9, 10) that the distribution of fringes depends on the parameters of the optical system. This is partly due to the nonrectilinear propagation of light.

Besides interference fringes on which the integral phase retardation is constant, on fringe patterns (Fig. 9), additional fringes are to be seen. These fringes must have a different physical origin.

At the end of the paper application of collimated and diffused light polariscopes is compared in respect to deflection of light rays. 